\title{
Wavelength calibration of Brewer spectrophotometer using a tunable pulsed laser and implications to the Brewer ozone retrieval
}

\author{
Alberto Redondas ${ }^{1,2}$, Saulius Nevas ${ }^{3}$, Alberto Berjón ${ }^{4,2}$, Meelis-Mait Sildoja ${ }^{3}$, Sergio Fabian León-Luis ${ }^{1,2}$, \\ Virgilio Carreño $^{1,2}$, and Daniel Santana-Díaz ${ }^{4,2}$ \\ ${ }^{1}$ Agencia Estatal de Meteorología, Izaña Atmospheric Research Center, Tenerife, Spain \\ ${ }^{2}$ Regional Brewer Calibration Center for Europe, Izaña Atmospheric Research Center, Tenerife, Spain \\ ${ }^{3}$ Physikalisch-Technische Bundesanstalt (PTB), Braunschweig, Germany \\ ${ }^{4}$ University of La Laguna, Department of Industrial Engineering, S.C. de Tenerife, Spain
}

Correspondence: Alberto Redondas (aredondasm@aemet.es)

Received: 15 December 2017 - Discussion started: 31 January 2018

Revised: 25 May 2018 - Accepted: 1 June 2018 - Published: 27 June 2018

\begin{abstract}
In this contribution we present the wavelength calibration of the travelling reference Brewer spectrometer of the Regional Brewer Calibration Center for Europe (RBCCE) at PTB in Braunschweig, Germany. The wavelength calibration is needed for the calculation of the ozone absorption coefficients used by the Brewer ozone algorithm. In order to validate the standard procedure for determining Brewer's wavelength scale, a calibration has been performed by using a tunable laser source at PTB in the framework of the EMRP project ENV59 ATMOZ "Traceability for the total column ozone". Here we compare these results to those of the standard procedure for the wavelength calibration of the Brewer instrument. Such a comparison allows validating the standard methodology used for measuring the ozone absorption coefficient with respect to several assumptions. The results of the laser-based calibrations reproduces those obtained by the standard operational methodology and shows that there is an underestimation of $0.8 \%$ of the ozone absorption coefficients due to the use of the parametrized slit functions.
\end{abstract}

\section{Background}

Nowadays the primary ground-based instruments used to report total ozone column (TOC) are Dobson and Brewer spectrophotometers. Based on the irradiances measured by these instruments in the ultraviolet (UV) spectral range and on well-defined retrieval procedures, TOC values are derived. The Brewer spectrometer (Brewer, 1973; Kerr et al.,
1981; Kerr, 2010) was introduced in the 1980s as an automatic device measuring direct solar UV radiation and global UV irradiance. Both the Brewer and the Dobson instruments were considered by the World Meteorological Organization (WMO) in the framework of the Global Atmosphere Watch program (GAW) as the standard instruments for TOC monitoring. The wavelength calibration is needed for calculation of the ozone absorption coefficient used by the Brewer ozone retrieval algorithm. The Brewer spectrophotometer has two operating modes. In the ozone mode, used for the total ozone column and aerosol measurements, the diffraction grating stays at a fixed position while the six operational wavelengths are selected by a rotating slit mask (Table 1). The scanning mode is used to perform spectral irradiance measurements in the UV spectral range. In this mode, the slits are fixed and the spectral scan is carried out by turning the diffraction grating. The usual wavelength calibration procedure is performed in the scanning mode by analyzing recorded emission lines of the spectral discharge lamps, which are usually mercury $(\mathrm{Hg})$, cadmium $(\mathrm{Cd})$, and zinc $(\mathrm{Zn})$. The use of the spectral lines provided in Table 2 allow us to determine the central wavelengths and the corresponding full-widthat-half-maximum (FWHM) of the slit functions as well as the relation between the positions of the grating and the corresponding instrument wavelengths (dispersion relation) required to determine the operational wavelengths used for the ozone determination. To obtain the ozone absorption coefficients, the instrumental slit functions are convolved with the 
Table 1. Slits and wavelengths used in the Brewer operative algorithms. The table provides the mean and the standard deviation of the central wavelengths and the full-width-half-maximum (FWHM) in nanometres of the slits of the "average" Brewer spectrophotometer determined during RBCC-E campaigns (Redondas et al., 2014).

\begin{tabular}{lrrrr}
\hline Slit & Wavelength & SD & FWHM & SD \\
\hline 0 & 306.301 & 0.014 & 0.548 & 0.016 \\
2 & 310.051 & 0.014 & 0.539 & 0.015 \\
3 & 313.501 & 0.015 & 0.555 & 0.012 \\
4 & 316.801 & 0.017 & 0.545 & 0.012 \\
5 & 320.002 & 0.019 & 0.538 & 0.012 \\
\hline
\end{tabular}

Bass \& Paur ozone absorption cross-section data. The use of a tunable laser source allows us to do the following:

- The calculation of the ozone absorption coefficients from the calibration directly in the ozone mode. The normal determination of the ozone absorption coefficients involves scanning of the spectral lines in the scanning mode of the instrument so that the dispersion relation is required to convert the grating positions in micrometre steps to the respective wavelengths. Here we can determine the instrumental slit functions directly in the ozone mode by scanning them with the tunable laser and weight them with the ozone absorption crosssections without the need of assumption about the slit functions and the dispersion relations used in the normal calibration procedure (See Sect. 2).

- The calculation of the dispersion relation based on regularly spaced reference spectral lines, provided by the tunable laser instead of the irregularly distributed emission lines of the $\mathrm{Hg}, \mathrm{Cd}$ and $\mathrm{Zn}$ spectral lamps.

During the experiment we performed three measurements:

1. The standard method for the dispersion measurements using spectral lamps described in Sect. 2.

2. Direct dispersion measurements (laser scanning). While the Brewer spectrophotometer is measuring in the ozone mode, the laser wavelength is swept $\pm 2 \mathrm{~nm}$ with a step of $0.04 \mathrm{~nm}$ around the six Brewer operational wavelengths selected by the rotating slit mask for different grating positions (Fig. 1)

3. Dispersion measurements using the tunable laser (Brewer scanning). While the laser is emitting at a fixed wavelength in the range from 290 to $365 \mathrm{~nm}$ with an increment of $5 \mathrm{~nm}$, the Brewer instrument scans $\pm 2 \mathrm{~nm}$ around these wavelengths in the scanning mode by moving the grating and using the six slits. The results allow us to estimate the dispersion approximation error due to the lack of spectral lines available from the discharge
Table 2. Emission lines of the discharge lamps used for Brewer calibration.

\begin{tabular}{lrr}
\hline Lamp & Line (nm) & Slits \\
\hline Mercury (Hg) & 289.36 & $0-1$ \\
$\mathrm{Hg}$ & 296.728 & $0-3$ \\
Zinc (Zn) & 301.836 & $0-5$ \\
Zn & 303.578 & $0-5$ \\
Cd (multiplet) & 308.082 & $0-5$ \\
$\mathrm{Cd}$ & 313.3167 & $0-5$ \\
$\mathrm{Cd}$ & 326.1055 & $0-5$ \\
$\mathrm{Zn}$ & 328.233 & $0-5$ \\
$\mathrm{Hg}$ & 334.148 & $0-5$ \\
$\mathrm{Cd}$ & 340.3652 & $0-5$ \\
$\mathrm{Cd}$ & 349.995 & $4-5$ \\
$\mathrm{Cd}$ (multiplet) & 361.163 & 5 \\
\hline
\end{tabular}

lamps at the end of spectral range of the Brewer spectrophotometer and due to the fact that the emission lines of the used lamps are not equally spaced.

\section{Calibration of the Brewer spectrophotometer}

The Brewer instrument measures the irradiance of direct sunlight at six nominal wavelengths $(\lambda)$ in the UV range: 303.2 , $306.3,310.1,313.5,316.8$ and $320.1 \mathrm{~nm}$, each spectral band covering a bandwidth of $0.5 \mathrm{~nm}$ (resolving power $\lambda / \delta \lambda$ of around 600). The spectral resolution is achieved by a holographic grating in combination with a slit mask that selects the channel to be analyzed by a photomultiplier tube (PMT). The longest four wavelengths are used for the ozone calculation. Based on the Lambert-Beer law, the total ozone column in the Brewer algorithm can be expressed as follows:

$X=\frac{F-\mathrm{ETC}}{\alpha \mu}$

where $F$ are the measured double ratios corrected for Rayleigh effects, $\alpha$ is the ozone absorption coefficient, $\mu$ is the ozone air mass factor, and ETC is the extra-terrestrial constant. The $F, \alpha$ and ETC parameters are weighted functions at the operational wavelengths with weighting coefficients $w_{i}$ :

$$
\begin{aligned}
& F=\sum_{i}^{4} w_{i} F_{i}-\frac{p}{p_{0}} \beta_{i} \mu, \\
& \alpha=\sum_{i}^{4} w_{i} \alpha_{i}, \\
& \mathrm{ETC}=\sum_{i}^{4} w_{i} F_{0 i},
\end{aligned}
$$

where, $\beta_{i}$ are the Rayleigh coefficients, $p$ is the climatological pressure at the measurement site, $p_{0}$ is the pressure at sea 

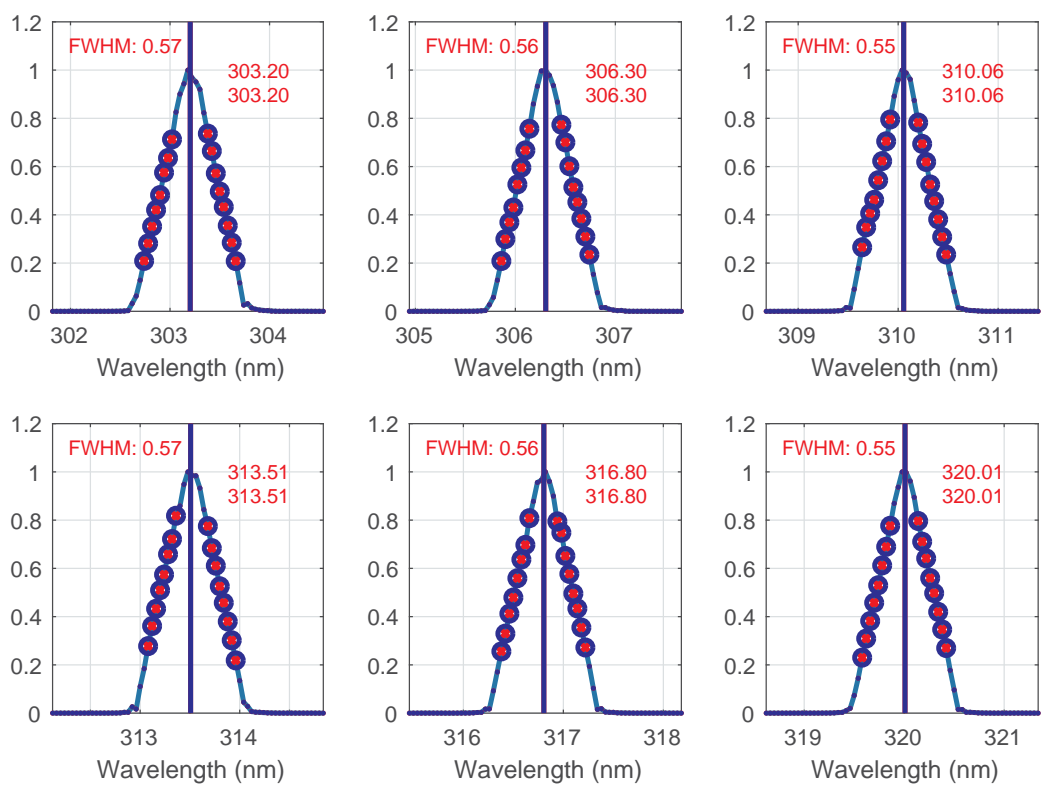

Figure 1. Results of Brewer measurements in the ozone mode obtained while the laser wavelength was changed every $0.04 \mathrm{~nm}$. The central wavelength and the FWHM calculated are displayed in red using the same methodology of the dispersion test.

level, and $F_{0}$ are the individual extra-terrestrial constants at each wavelength. The weights $w=[1,-0.5,-2.2,1.7]$ have been chosen to minimize the influence of $\mathrm{SO}_{2}$ and verify the following:

$$
\begin{aligned}
& \sum_{i}^{4} w_{i}=0, \\
& \sum_{i}^{4} w_{i} \lambda_{i}=0 .
\end{aligned}
$$

This widely eliminates absorption features which depend, in local approximation, linearly on the wavelength, like for example the contribution from aerosols.

We can divide the calibration in three steps including instrumental calibration, wavelength calibration, and ETC transfer:

1. The instrumental calibration includes all the parameters that affect the double ratios $(F)$ obtained from the signals measured with the different slits in front of the PMT, in particular, photomultiplier dead time correction, temperature coefficients and filter attenuation.

2. Wavelength calibration is needed to determine the ozone absorption coefficient. The so-called "dispersion test" is used to obtain the particular wavelengths for the instrument and the slits, or instrumental functions, of each spectrophotometer. Note that the precise wavelengths of every Brewer spectrophotometer are slightly different from instrument to instrument.
3. Finally, the ETC transfer is performed by comparison with the reference Brewer instrument or, in the case of the reference instruments, by the Langley method.

The Brewer wavelength calibration follows the operative procedure (Gröbner et al., 1998; Kerr, 2002) used by the Regional Brewer Calibration Center for Europe (RBCC-E) at the calibration campaigns. In summary, the individual wavelengths (bands) in the Brewer instrument are selected through the use of a stainless steel mask of seven slits located at the focal plane of the spectrometer. The particular wavelength is determined by analyzing the measurements of a series of discharge lamps during so-called dispersion test, which determines the central wavelength and FWHM of every slit. Then the wavelength setting is optimized to minimize the effect of wavelength shift during the operation of the instrument by performing the so-called sun-scan test (Lamb and Asbridge, 1996). Finally, the ozone absorption coefficient is determined for every slit.

The ozone absorption coefficient were defined as follows (Vanier and Wardle, 1969; Bernhard et al., 2005):

$\alpha_{i}(\lambda)=\frac{\log \left(\frac{\int E_{o}(\lambda) S\left(\lambda, \lambda_{i}\right) 10^{-\sigma(\lambda) X \mu-\beta(\lambda) \frac{P}{P_{o}} \nu} \mathrm{d} \lambda}{\int E_{o}(\lambda) S\left(\lambda, \lambda_{i}\right) 10^{-\beta(\lambda) \frac{P}{P_{o}} v} \mathrm{~d} \lambda}\right)}{X \mu}$,

where $S$ is the instrumental slit function for the corresponding wavelength, $E$ is the sun spectrum at this wavelength that depends mostly on the ozone concentration and air-mass, and $\sigma$ is the ozone cross-section at the temperature of $-46.3{ }^{\circ} \mathrm{C}$ for Dobson and at $-45^{\circ} \mathrm{C}$ for Brewer instruments. 
The Brewer operative method uses the following assumptions:

1. Only "ideal" slits are used; the slit functions are parametrized as trapezoids, i.e. isosceles triangles truncated at 0.87 height.

2. Stray light is not considered, i.e. zero slit function values are assumed outside the triangle.

3. The FWHM of the triangle is considered different for every slit. It is calculated from the dispersion test, determined in micrometre steps and then converted to wavelengths using the dispersion relation (Fig. 2).

4. The ozone cross sections are expressed by the Bass \& Paur absorption coefficient data set.

5. Solar spectrum is considered constant on the slit.

Under these assumptions, the ozone effective absorption is essentially obtained the same way as in the approximation method of Bernhard et al. (2005) used with Dobson spectrophotometers (see Eq. 8).

$\alpha_{i}=\frac{\int \sigma(\lambda) S_{i}\left(\lambda, \lambda^{\prime}\right) \mathrm{d} \lambda^{\prime}}{\int S_{i}\left(\lambda, \lambda^{\prime}\right) \mathrm{d} \lambda^{\prime}}$

\section{Dispersion Test}

The Brewer spectrophotometer is constructed based either on a single or a double monochromator of modified EbertFastie type, generally referred to as single or double Brewer, respectively. The first monochromator disperses the incoming radiation onto six exit slits. In the case of the doublemonochromator Brewer, the six exit slits (intermediate slits) of the first monochromator are the entrance slits to a second monochromator that is used in subtractive mode. The wavelength is selected by choosing one of the six exit slits (ozone mode) or rotating the grating (scanning mode). The rotation of the grating is managed by a drive mechanism consisting of a motor-driven micrometre linked to an arm that rotates the grating. The smallest wavelength increment corresponding to one stepper motor step varies steadily from approximately 8.0 to $7.0 \mathrm{pm}$ (Gröbner et al., 1998).

The dispersion relation, which provides the relation between the micrometre steps and the monochromator set wavelengths, is determined by scanning the emission lines as described in Sect. 1. The line scans are carried out with an increment of 10 motor steps $(0.7 \AA)$. From the results, the central position and the FWHM of the slit function are calculated in motor steps assuming an isosceles triangle. Both sides of the peak are fitted to a straight line taking only the function values above 20 and below $80 \%$ of the normalized peak. The central point is calculated by the intersection point and the FWHM is the width of the triangle (Fig. 2). Finally, the dispersion relation is calculated using a quadratic polynomial or the cubic polynomial approximation. This relation

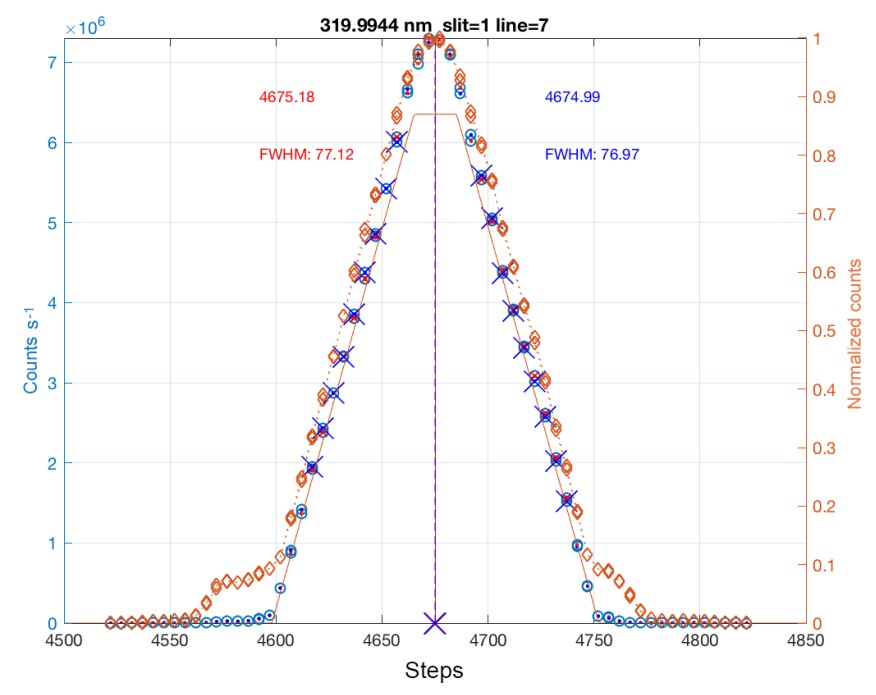

Figure 2. Slit function of the slit $3(320 \mathrm{~nm})$ measured in the scanning mode: the standard method uses the normalized values only between 0.2 and 0.8 (points with crosses). The figure shows the signals recorded during the scans (orange diamonds) and the nonlinearity-corrected values (blue circles) in counts $\mathrm{s}^{-1}$. The hysteresis is evident from the asymmetry of the uncorrected signals in the low signal region of the plot. The centre steps and the FWHMs calculated from the up-scan are shown in red while the values derived from the down-scanned slit function are presented in blue text. The central wavelength determination is not affected by the nonlinearity but the apparent FWHMs would be larger if the non-linearity correction were not applied. The resulting parametrized slits are represented on the right axis.

is used to transform the previously determined central positions and FWHMs of the slit functions in micrometre steps to a wavelength scale.

The cubic approximation method of Gröbner et al. (1998) uses knowledge of the optical design of the Brewer spectrometer to transfer results of the spectral line measurements from one slit to the other slits, which reduces the number of free parameters to be adjusted compared with the quadratic method. However, there is a systematic difference between the ozone absorption coefficients calculated by the two methods. Both methods generally agree only in the ozone spectral range of the Brewer instrument (Redondas and RodriguezFranco, 2014).

The stability of the wavelength calibration during Brewer operations is checked by measuring the internal $\mathrm{Hg}$ lamp. In most of the Brewers, the $302 \mathrm{~nm}$ double line (302.150 and $302.347 \mathrm{~nm}$ ) is used due to its proximity to the Brewer operational wavelengths. However, for Brewer \#185 and for an increasing number of other Brewers the test is performed using the more powerful $296.7 \mathrm{~nm}$ line. The wavelength test includes 12 measurements of the line from the mercury lamp on slit 0 , with an increment of \pm 10 steps of the micrometre motor. The obtained curve for the line peak is compared to a stored reference one. The comparison is done by shifting 


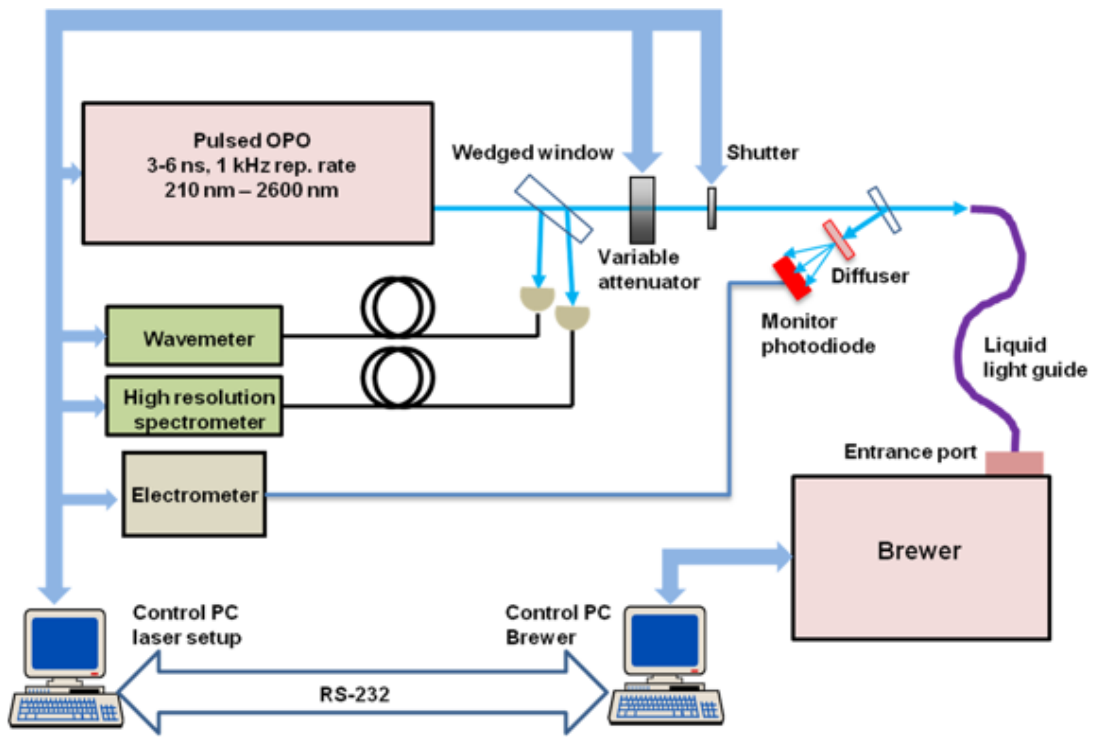

Figure 3. Pulsed OPO-based setup at PTB that was used for measuring the slit functions of the Brewer spectrophotometer.

the two scanned curves against each other and calculating the correlation coefficient between the two after each shift. The interpolated step number yielding the maximum of the correlation coefficient provides the reference micrometre position (Savastiouk, 2006). If the required adjustment of the micrometre position is more than one and a half motor steps, the test is repeated. Hence, the accuracy of the wavelength setting of the Brewer instrument achieved by such an approach is defined by a rectangular probability distribution function on the interval of \pm 0.5 steps. Thus, the respective standard uncertainty is $0.5 / \sqrt{3}=0.289$ steps. During the brewer operation at RBCC-E calibration campaigns the ozone observations are discarded if the subsequent hg test is repeated, the bigger discrepancy for the accepted measurements will be 1.5 steps. This 1.5 affects the ozone absorption coefficient by approximately of $0.1 \mathrm{~atm} \mathrm{~cm}^{-1}$ and the ozone concentration by $0.3 \%$.

\section{Pulsed laser-based measurements}

\subsection{Instrumental setup}

For the characterization of the bandpass functions of the Brewer instrument, an upgraded PLACOS setup (Nevas et al., 2009) featuring a tunable pulsed laser system based on an optical parametric oscillator (OPO) was used at Physikalisch-Technische Bundesanstalt (PTB) in Braunschweig (Fig. 3). The new OPO system generates 3-6ns pulses at $1 \mathrm{kHz}$ repetition rate in the spectral range from 210 to $2600 \mathrm{~nm}$. The laser beam was guided into the direct port of the Brewer spectrophotometer by using a liquid light guide. A fraction of the beam was directed to a monitor photodiode in order to account for the output power changes of the laser

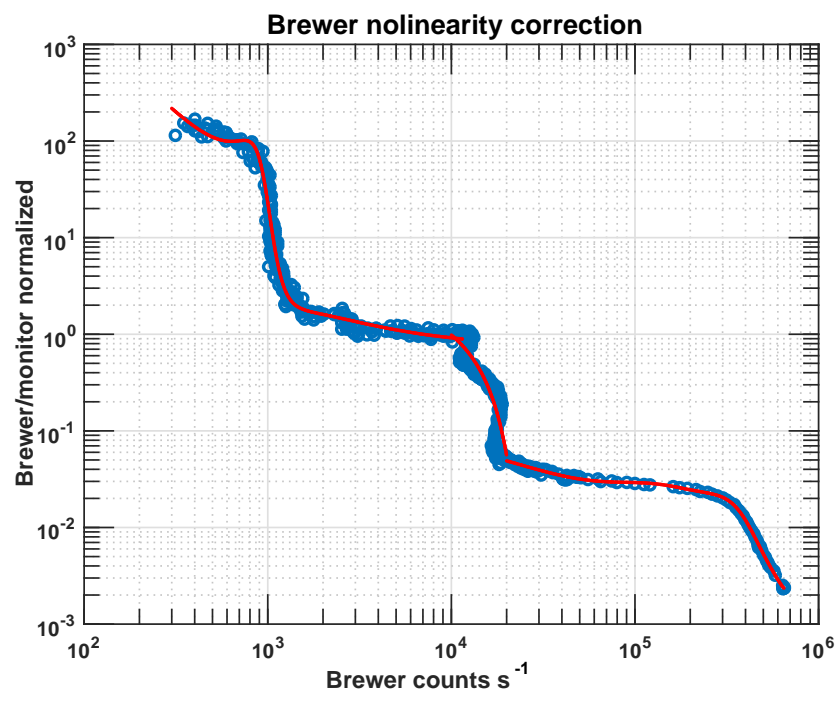

Figure 4. Log-log plot of the normalized ratio of measured Brewer counts to the monitor photodiode signal, which is proportional to the laser power, plotted as a function of the Brewer counts $\mathrm{s}^{-1}$. The black points shows the measured points while the red curve is the fit used to correct the Brewer signals for the nonlinearity.

beam. The photocurrent of the silicon photodiode was measured by an electrometer. During the measurements, the laser wavelengths were monitored by a wavemetre (laser spectrum analyzer) and a high-resolution spectrometer with a FWHM of $0.1 \mathrm{~nm}$. The wavelength accuracy of the wavemetre according to the manufacturer's specifications is between 6 and $3 \mathrm{pm}$ in the spectral range from 290 to $360 \mathrm{~nm}$, respectively. The calibration of the instrument is accomplished by means of an autocalibration procedure based on an internally built- 


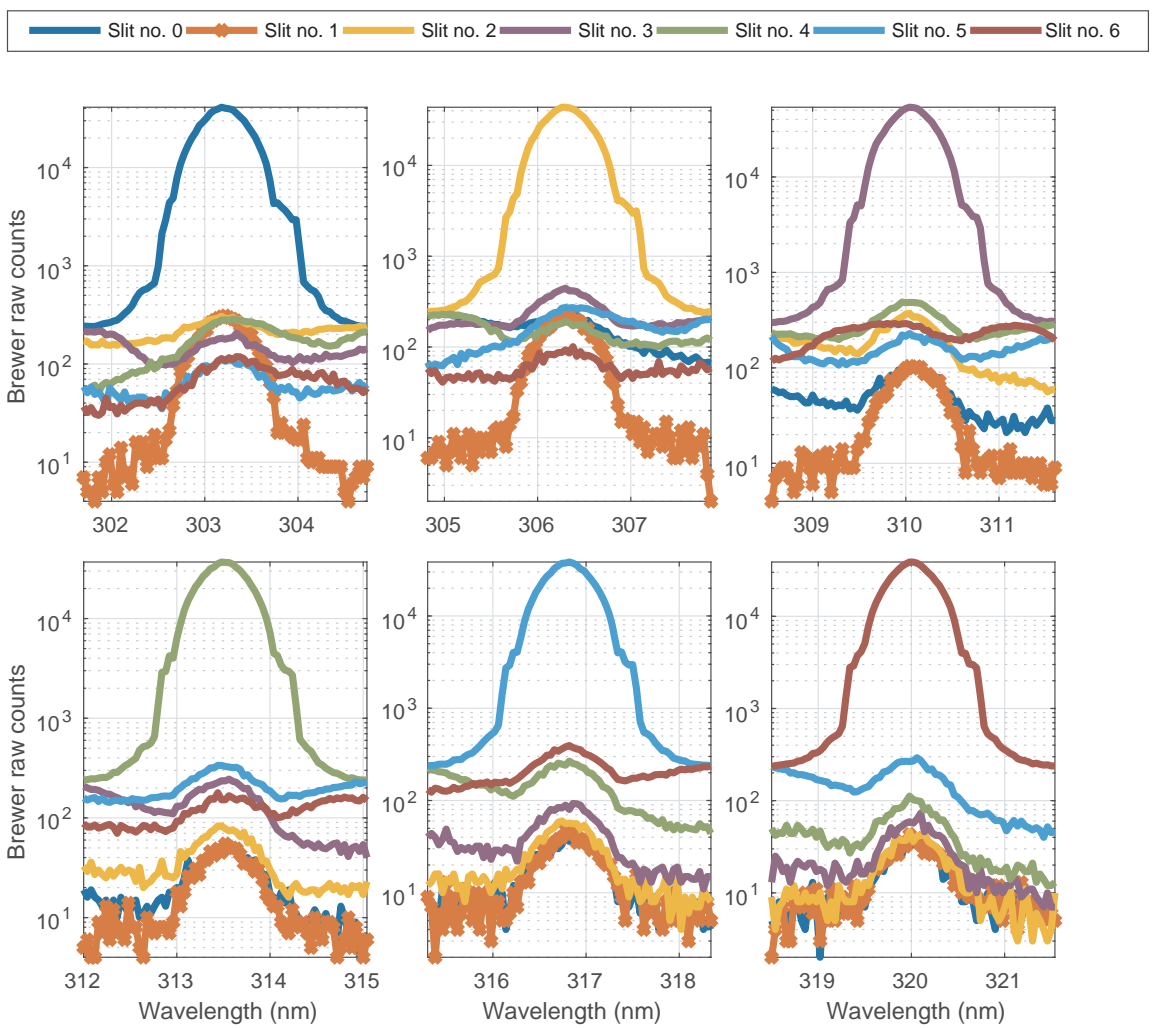

Figure 5. Brewer measurements in the ozone mode while the laser wavelength is changed every $0.04 \mathrm{~nm}$. The orange curve corresponds to the dark counts obtained from the measurements of slit 1 .

in neon lamp. The wavelength scale of the high-resolution spectrometer was calibrated by using the spectral emission lines of a Hg lamp. The wavelength uncertainty of the calibrated spectrometer in the above-mentioned spectral range was estimated to be $10 \mathrm{pm}$. A side-by-side comparison of the laser wavelength values measured by both instrument types showed an agreement well within $10 \mathrm{pm}$.

In contrast with the standard calibration procedure, where the Brewer instrument scans the lines of the spectral lamps, in this experiment the Brewer measures in ozone mode. Here, the Brewer grating is fixed at the ozone position while the coupled laser beam is measured using the seven slits (slit \#1 is used to obtain the dark signal values). During these measurements the wavelength of the OPO system is scanned with $0.04 \mathrm{~nm}$ step. The experiment is complemented by the measurements in the Brewer scanning mode, where the tunable laser is used as a source of spectral lines covering the range from 290 to $360 \mathrm{~nm}$ on a regular grid with $5 \mathrm{~nm}$ step.

\subsubsection{Non-linearity of the PMT}

The Brewer detector system, which is based on a PMT, responds non-linearly to pulsed sources. For the measurements of pulsed sources, the PMT manual advises changing the electronics configuration. As the main objective was to validate the operational wavelength calibration of the Brewer, we decided to keep the instrument configuration equivalent to that during the field operations. The non-linearity problem was solved by determining the respective correction function. For this purpose, the power of the laser beam was varied while simultaneously measuring signals of the PMT and the linear monitor photodiode.

The ratios of the measured Brewer counts to the recorded monitor photodiode signals are shown in Fig. 4. The nonlinearity is evident together with hysteresis region near $10^{4}$ Brewer counts $\mathrm{s}^{-1}$. The correction is not reliable around $10^{3}$ counts s$^{-1}$ and lower than 100 counts s$^{-1}$. As we can control the power of the laser beam, it is possible to work on the "flat regions" of the non-linearity characteristics and apply the determined correction. This correction does not affect the calculated central wavelength of the slit functions, though, it does affect the determined FWHM values (Fig. 2) if the correction is not applied.

We observed that the recorder dark signal values (measurements performed with the blocked slit \#1) were highest 

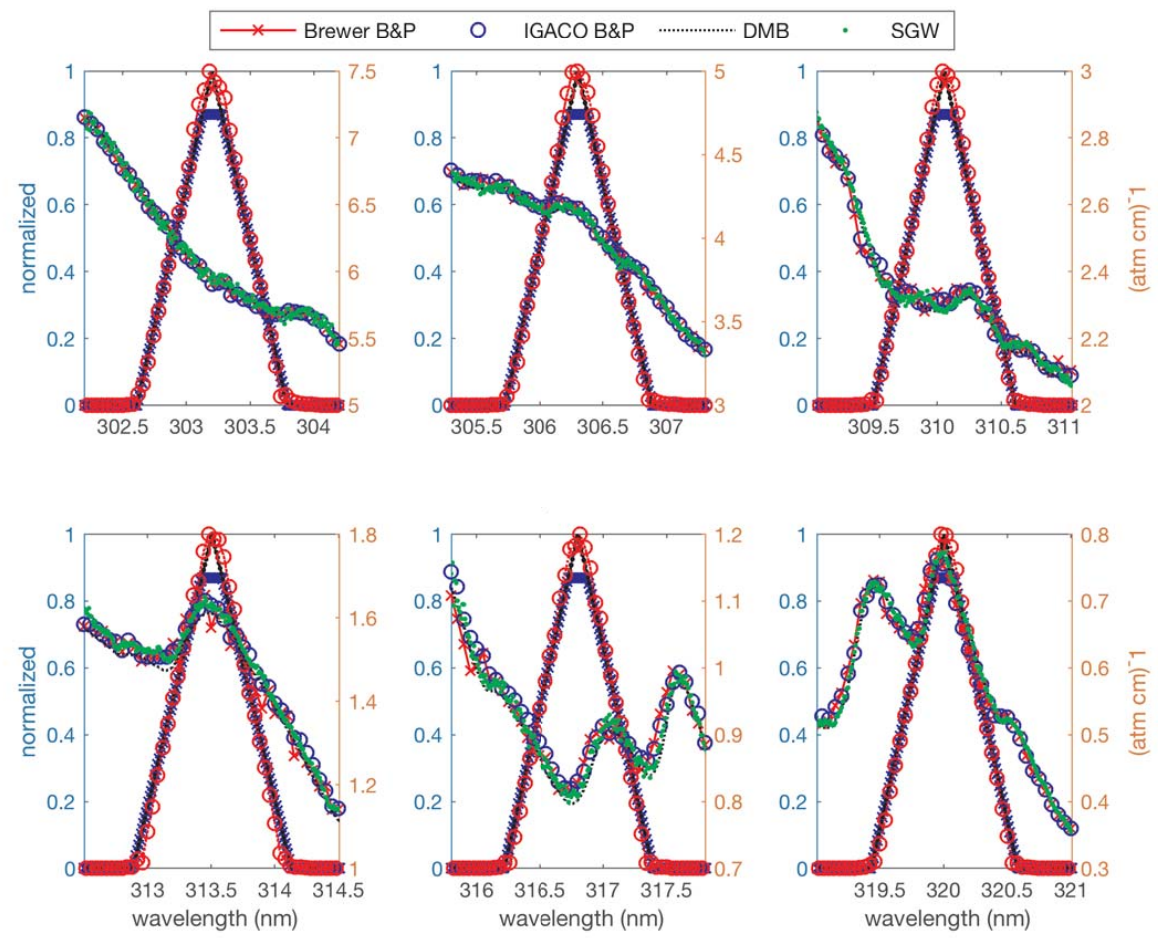

Figure 6. Plot of the parametrized (thick lines, left axis) and the measured slit functions (dots, left axis), as well as the different ozone cross-sections in $\mathrm{cm}^{-1}$ (right axis) used for the Brewer effective ozone absorption coefficient calculation.

Table 3. Ozone absorption coefficients in atm $\mathrm{cm}^{-1}$ calculated using four ozone absorption cross-sections, together with the trapezoidal and the triangular parametrization of the slit functions, as well as the directly measured slit functions. Percentage difference is also given between the absorption coefficients yielded by the parameterized and the directly measured slit functions.

\begin{tabular}{lrrrrr}
\hline & Trapezoid & Triangular & Measured & \% Trapezoid & \% Triangular \\
\hline BRW & 0.3381 & 0.3395 & 0.3406 & -0.73 & -0.33 \\
B\&P & 0.3331 & 0.3344 & 0.3359 & -0.85 & -0.44 \\
DMB & 0.3483 & 0.3498 & 0.3514 & -0.86 & -0.45 \\
SGW & 0.3393 & 0.3407 & 0.3422 & -0.84 & -0.43 \\
\hline
\end{tabular}

immediately after exposing the PMT to the laser light (see Fig. 5). The dark signal of the PMT was then gradually fast decreasing with time after the excitation, which may cause the signal values obtained for slit \#1 (measured immediately after slit \#0) be higher than for the other slits measured afterwards.

\subsubsection{Slit parametrization}

The Brewer algorithms assume trapezoidal slits functions cut at 0.87 of the height (Fig. 6) with the centre wavelength and the FWHM calculated for every slit from the dispersion relation. The laser measurements allow us to estimate the effect on the ozone calculation if we use the directly measured slit functions instead of the parametrized ones. For this purpose we calculate the ozone absorption coefficients for the four ozone cross sections evaluated in the "ACSO" commit- tee ("Absorption Cross Sections of Ozone") (Orphal et al., 2016).

Among the available data sets there are versions of Bass and Paur (1985) cross-sections denoted as Brewer operational (Brw), IGACO quadratic coefficient (B\&P), the crosssections of Daumont Brion Malicet (DBM) (Daumont et al., 1992; Brion et al., 1993; Malicet et al., 1995), and the newly recommended data set for ozone ground-based calculation by Serdyuchenco, Groshelev, Weber (SGW) (Serdyuchenko et al., 2012; Gorshelev et al., 2014; Weber et al., 2016).

Using the measured slit functions, the calculated effective ozone cross sections are $\sim 0.85 \%$ higher compared to those obtained by using the parametrized Brewer slits in the standard procedure (Table 3 ), this difference is reduced approximately by a half when a triangular parametrization is used $(0.44 \%)$, the results are very similar for all the cross sections, 

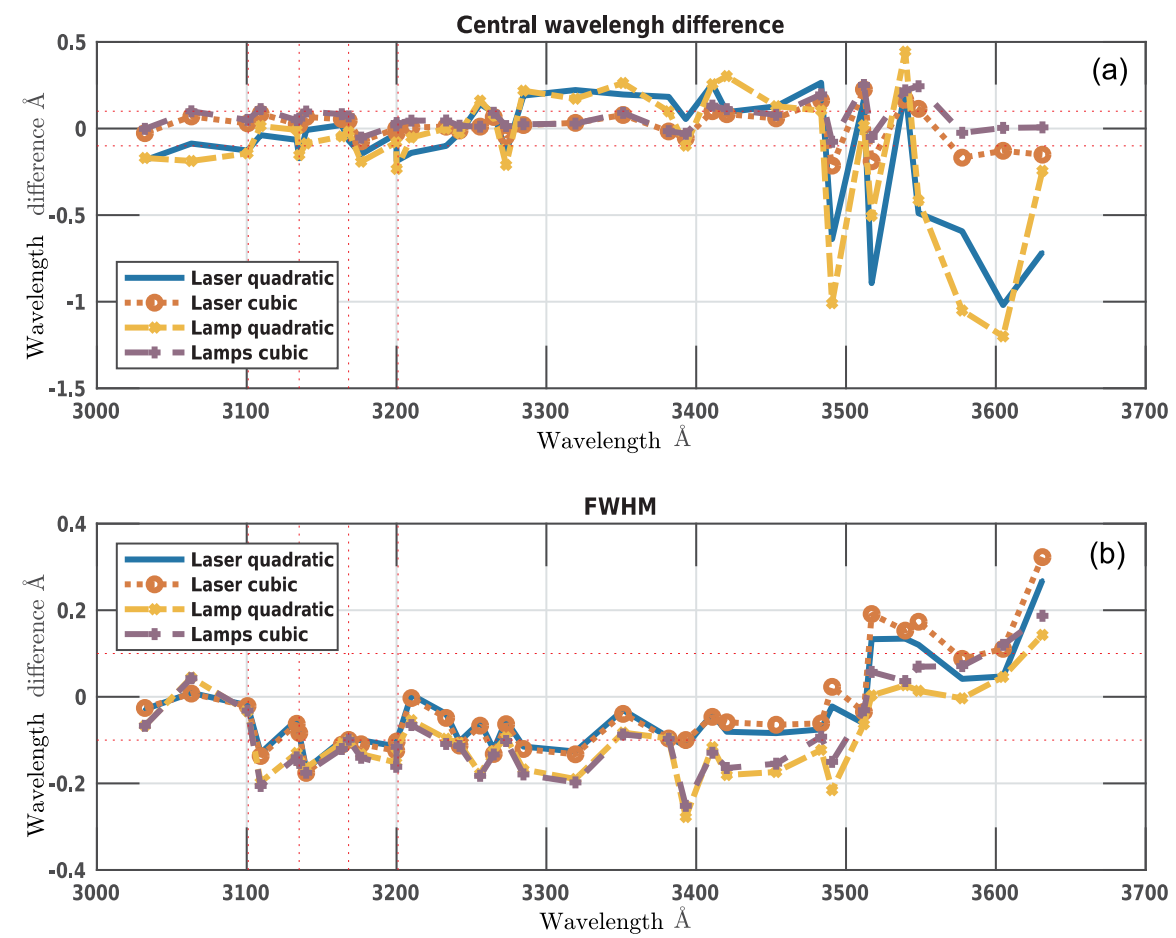

Figure 7. Differences between the central wavelengths (a) and FWHMs (b) determined directly and by the scanning methods, with the laser wavelengths in equally spaced grid every $5 \mathrm{~nm}$ and lines of the discharge lamps. In both cases quadratic and cubic fits are used.

Table 4. Ozone absorption coefficient, in atm $\mathrm{cm}^{-1}$, calculated using four absorption cross sections.

\begin{tabular}{|c|c|c|c|c|c|}
\hline & brw_scan ${ }^{\mathrm{a}}$ & opo_quad ${ }^{b}$ & opo_cubic ${ }^{c}$ & lamp_quad ${ }^{\mathrm{d}}$ & lamp_cubic ${ }^{\mathrm{e}}$ \\
\hline SGW & 0.3409 & 0.3442 & 0.342 & 0.3446 & 0.3412 \\
\hline Ratio & 1 & 0.9881 & 1.0033 & 1.0108 & 1.001 \\
\hline
\end{tabular}

with the exception of the Bass \& Paur used by the Brewer algorithm due its low resolution.

\section{Discussion}

The experiment allows us to validate the Brewer standard methodology used to perform the wavelength calibration. For this purpose, we compare laser-based wavelength calibration results to those yielded by the standard operative method based on scanning the spectral lamps in case of both the quadratic and the cubic fit to the dispersion relation.

Figure 7 shows discrepancies between the central wavelengths calculated by the quadratic and the cubic fits to be bigger than $0.1 \AA$ for wavelength above $320 \mathrm{~nm}$ and much bigger near $350 \mathrm{~nm}$. This is also indicated by a systematic shape of the residuals of the quadratic fit that are much larger than for the cubic fit (Fig. 8). This indicates that the quadratic fit is only valid in the ozone range $(310-320 \mathrm{~nm})$ as already noted in Gröbner et al. (1998). The comparison of the calculated FWHMs (Fig. 7) shows a different pattern with a difference of $0.1 \AA$ between the direct and the scanning methods in the ozone range and with a smaller difference between the quadratic and the cubic fits.

The differences between the ozone absorption coefficients calculated from the scanning and the direct measurements of the slit functions are summarized for the six measurements in Table 4, taking the direct measurements as a reference. The quadratic fits result in bigger differences of around $1 \%$, whereas in the case of the cubic fits the differences decrease to 0.3 and $0.1 \%$ when the laser or the discharge lamps are used, respectively. 


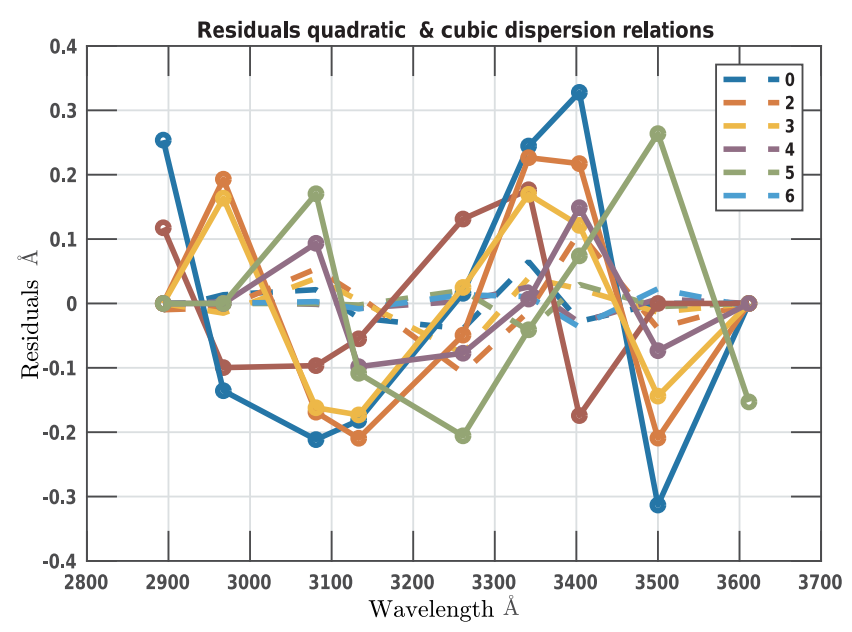

Figure 8. Residuals of the quadratic (filled circles, solid lines) and cubic (dashed lines) fits. The colour indicates the six Brewer slits with the laser wavelengths in equally spaced grid every $5 \mathrm{~nm}$.

\section{Conclusions}

1. Using the measured slit functions instead of the parametrized ones increases the ozone absorption coefficients and consequently the calculated ozone values by $0.8 \%$.

2. The quadratic dispersion relation fit used in the standard Brewer algorithm is not suitable outside the ozone spectral range $310-320 \mathrm{~nm}$. The residuals show a systematic pattern, which is particularly important at the upper end of the spectral range.

3. The comparison of the results of the three experiments shows a maximum difference of $0.3 \%$ if the cubic fit is used to approximate the dispersion relation of the Brewer instrument. The respective difference between the ozone absorption coefficient that is obtained from the direct measurements of the tunable laser in the ozone mode and from the operative discharge lamp method is only of $0.1 \%$, if both use the parametrized or measured slit. This confirms the standard procedure used for the RBCC-E calibrations.

4. This work validates the current wavelength calibration method of the Brewer network and shows that in general there is no need for the characterization of the network Brewer instruments with a tuneable source. However, the limitations of the quadratic dispersion approximation used over extended spectral range of Brewers MKIII and MK-IV are evident. Thus, we suggest updating the operating Brewer software for this model instruments with the recent version, which already includes the cubic dispersion.
Data availability. The data used for the present study can be made available after personal communication with the authors of the paper.

Competing interests. The authors declare that they have no conflict of interest.

Special issue statement. This article is part of the special issue "Quadrennial Ozone Symposium 2016 - Status and trends of atmospheric ozone (ACP/AMT inter-journal SI)". It is a result of the Quadrennial Ozone Symposium 2016, Edinburgh, United Kingdom, 4-9 September 2016.

Acknowledgements. This work has been supported by the European Metrology Research Programme (EMRP) within the joint research project ENV59 "Traceability for atmospheric total column ozone" (ATMOZ). The EMRP is jointly funded by the EMRP participating countries within EURAMET and the European Union.

Edited by: Alkiviadis Bais

Reviewed by: Lionel Doppler and Julian Gröbner

\section{References}

Bernhard, G., Evans, R. D., Labow, G. J., and Oltmans, S. J.: Bias in Dobson total ozone measurements at high latitudes due to approximations in calculations of ozone absorption coefficients and air mass, J. Geophys. Res.-Atmos., 110, D10305, https://doi.org/10.1029/2004JD005559, 2005.

Brewer, A. W.: A replacement for the Dobson spectrophotometer?, Pure Appl. Geophys., 106, 919-927, https://doi.org/10.1007/BF00881042, 1973.

Brion, J., Chakir, A., Daumont, D., Malicet, J., and Parisse, C.: High-resolution laboratory absorption cross section of $\mathrm{O}_{3}$. Temperature effect, Chem. Phys. Lett., 213, 610-612, https://doi.org/10.1016/0009-2614(93)89169-I, 1993.

Daumont, Brion, J., Charbonnier, J., and Malicet, J.: Ozone UV spectroscopy I: Absorption cross-sections at room temperature, J. Atmos. Chem., 15, 145-155, https://doi.org/10.1007/BF00053756, 1992.

Gorshelev, V., Serdyuchenko, A., Weber, M., Chehade, W., and Burrows, J. P.: High spectral resolution ozone absorption crosssections - Part 1: Measurements, data analysis and comparison with previous measurements around $293 \mathrm{~K}$, Atmos. Meas. Tech., 7, 609-624, https://doi.org/10.5194/amt-7-609-2014, 2014.

Gröbner, J., Wardle, D. I., McElroy, C. T., and Kerr, J. B.: Investigation of the wavelength accuracy of Brewer spectrophotometers, Appl. Optics, 37, 8352-8360, https://doi.org/10.1364/AO.37.008352, 1998.

Kerr, J.: New methodology for deriving total ozone and other atmospheric variables from Brewer spectrophotometer direct sun spectra, J. Geophys. Res.-Atmos., 107, ACH 22-1-ACH 22-17, https://doi.org/10.1029/2001JD001227, 2002. 
Kerr, J. B.: The Brewer Spectrophotometer, in: UV Radiation in Global Climate Change, edited by: Gao, W., Slusser, J. R., and Schmoldt, D. L., 160-191, Springer, Berlin Heidelberg, 2010.

Kerr, J. B., McElroy, C. T., and Olafson, R. A.: Measurements of ozone with the Brewer ozone spectrophotometer, in: Proceedings of the Quadrennial Ozone Symposium held in Boulder, Colorado, August 1980, 74-79, J. London, 1981.

Lamb, K. and Asbridge, A. I.: Brewer Technical Note No.96.01 Sun scans and relationship to the wavelength calibration step number, Tech. rep., International Ozone Services, 1996.

Malicet, Daumont, D., Charbonnier, J., Parisse, C., Chakir, A., and Brion, J.: Ozone UV spectroscopy. II. Absorption cross-sections and temperature dependence, J. Atmos. Chem., 21, 263-273, https://doi.org/10.1007/BF00696758, 1995.

Nevas, S., Lindemann, M., Sperling, A., Teuber, A., and Maass, R.: Colorimetry of LEDs with array spectroradiometers, Mapan, 24, 153-162, 2009.

Orphal, J., Staehelin, J., Tamminen, J., Braathen, G., De Backer, M.-R., Bais, A., Balis, D., Barbe, A., Bhartia, P. K., Birk, M., Burkholder, J. B., Chance, K., von Clarmann, T., Cox, A., Degenstein, D., Evans, R., Flaud, J. M., Flittner, D., GodinBeekmann, S., Gorshelev, V., Gratien, A., Hare, E., Janssen, C., Kyrölä, E., McElroy, T., McPeters, R., Pastel, M., Petersen, M., Petropavlovskikh, I., Picquet-Varrault, B., Pitts, M., Labow, G., Rotger-Languereau, M., Leblanc, T., Lerot, C., Liu, X., Moussay, P., Redondas, A., Van Roozendael, M., Sander, S. P., Schneider, M., Serdyuchenko, A., Veefkind, P., Viallon, J., Viatte, C., Wagner, G., Weber, M., Wielgosz, R. I., and Zehner, C.: Absorption cross-sections of ozone in the ultraviolet and visible spectral regions: Status report 2015, J. Mol. Spectrosc., 327, 105-121, 2016.
Redondas, A. and Rodriguez-Franco, J. J.: Regional Brewer Calibration Center Europe CEOS campaigns: Instrumental Findings, in: Quadrennial International Ozone Symposium, 14th WMOGAW Brewer Users Group Meeting EUBREWNET \& WMO GAW, 2014.

Redondas, A., Evans, R., Stuebi, R., Köhler, U., and Weber, M.: Evaluation of the use of five laboratory-determined ozone absorption cross sections in Brewer and Dobson retrieval algorithms, Atmos. Chem. Phys., 14, 1635-1648, https://doi.org/10.5194/acp-14-1635-2014, 2014.

Savastiouk, V.: Improvements to the direct-sun ozone observations taken with the Brewer spectrophotometer, $\mathrm{PhD}$ thesis, York University, Toronto, Ontario, Canada, 44-57, available at: https://www.esrl.noaa.gov/gmd/grad/neubrew/docs/ publications/VladimirSavastiouk_PhD_thesis.pdf (last access: 20 June 2018), 2006.

Serdyuchenko, A., Gorshelev, V., Weber, M., Chehade, W., and Burrows, J. P.: High spectral resolution ozone absorption crosssections - Part 2: Temperature dependence, Atmos. Meas. Tech., 7, 625-636, https://doi.org/10.5194/amt-7-625-2014, 2014.

Vanier, J. and Wardle, D.: The effects of spectral resolution on total ozone measurements, Q. J. Roy. Meteor. Soc., 95, 395-399 1969.

Weber, M., Gorshelev, V., and Serdyuchenko, A.: Uncertainty budgets of major ozone absorption cross sections used in UV remote sensing applications, Atmos. Meas. Tech., 9, 4459-4470, https://doi.org/10.5194/amt-9-4459-2016, 2016. 